\title{
Correlação da ventilação voluntária máxima com a força e resistência dos músculos respiratórios em jovens
}

\author{
Correlation of maximum voluntary ventilation \\ to the strength and resistance of young \\ respiratory muscles
}

\author{
Francisco Tiago Oliveira de Oliveira1 (1) \\ Juliana Guimarães ${ }^{2}$ (1) \\ Jessica Ramos Ribeiro ${ }^{3}$ (1) \\ Igor Alonso Andrade de Oliveira 4 (1) \\ Felipe Augusto dos Santos Costa ${ }^{5}$ (1) \\ Celso Nascimento de Almeida 6 (1) \\ Cristiane Maria Carvalho Costa Dias ${ }^{7}$ (1)
}

\footnotetext{
'Autor para correspondência. Escola Bahiana de Medicina e Saúde Pública (Salvador). Bahia, Brasil. franciscooliveira@bahiana.edu.br ${ }^{2-7}$ Escola Bahiana de Medicina e Saúde Pública (Salvador). Bahia, Brasil. julianaguimaraess.f@gmail.com, jessicarfisio526@gmail.com, igoroliveira13.1@bahiana.edu.br, felipecosta16.1@bahiana.edu.br, celsoalmeida.pos@bahiana.edu.br, cmccdias@bahiana.edu.br
}

RESUMO | INTRODUÇãO: A ventilação voluntária máxima é um dos testes difundidos para avaliação da resistência da musculatura respiratória, mesmo sem ser validado para este fim. Na literatura ainda são encontradas controvérsias quanto a interpretação e aplicabilidade do uso da VVM na prática clínica. OBJETIVO: Verificar a correlação entre a ventilação voluntária máxima e a força e resistência dos músculos respiratórios em jovens hígidos. MATERIAIS E MÉTODOS: Estudo observacional de corte transversal realizado na Clínica. Foram incluídos indivíduos $>18$ anos, de ambos os sexos e hígidos. Os participantes tiveram sua avaliação da força muscular respiratória através do manovacuômetro, no qual se obteve a Pimáx e Pemáx. A resistência foi avaliada através do teste de carga constante pelo Power Breathe, utilizando $60 \%$ da Pimáx. A ventilação voluntária máxima foi realizada pelo espirômetro. Para a correlação das variáveis Pimáx, Pemáx e VVM foi aplicado o teste de correlação de Pearson. O estudo foi aprovado pelo comitê de ética, CAAE 10849519.9.0000.5544. RESULTADOS: Foram avaliados 27 participantes, em que 59,3\% eram do sexo masculino e 55,6\% ativos. A ventilação voluntária máxima com a Pimáx e Pemáx, apresentaram respectivamente $p=0,04$ e 0,02 e $r=0,53$ e 0,57. CONCLUSÃO: O teste de ventilação voluntária máxima possui uma correlação moderada com a força muscular respiratória, e não obtém correlação com o teste de carga constante.

PALAVRAS-CHAVE: Músculos respiratórios. Ventilação voluntária máxima. Voluntários saudáveis.
ABSTRACT | INTRODUCTION: Maximum voluntary ventilation is one of the widespread tests for assessing respiratory muscle strength, even without being validated for this purpose. Controversies are still found in the literature regarding the interpretation and applicability of the use of MVV in clinical practice. OBJECTIVE: To verify the correlation between maximum voluntary ventilation and respiratory muscle strength and endurance in healthy youngsters. MATERIALS AND METHODS: Observational cross-sectional study conducted at the Clinic. Individuals> 18 years of age, of both sexes and healthy were included. Participants had their respiratory muscle strength assessment using a manovacuometer, in which Pimax and Pmax were obtained. The resistance was evaluated through the constant load test by Power Breathe, using $60 \%$ of the Pimáx. Maximum voluntary ventilation was performed by a spirometer. Pearson's correlation test was applied to correlate the variables Pimax, Pmax and VVM. The study was approved by the ethics committee, CAAE 10849519.9.0000.5544. RESULTS: 27 participants were evaluated, of which $59.3 \%$ were male and $55.6 \%$ were active. The maximum voluntary ventilation with Pimax and Pmax, presented respectively $p=0.04$ and 0.02 and $r=0.53$ and 0.57 . CONCLUSION: The maximum voluntary ventilation test has a moderate correlation with respiratory muscle strength and has no correlation with the constant load test.

KEYWORDS: Respiratory muscles. Maximum voluntary ventilation. Healthy volunteers. 


\section{Introdução}

A avaliação do desempenho da musculatura respiratória e do sistema respiratório envolve testes para avaliação tanto da força, quanto da resistência dos músculos respiratórios, bem como da função pulmonar. Estas medidas guiam estratégias terapêuticas em programas de reabilitação cardiopulmonar e no ambiente de alto desempenho $0^{1,2}$.

Dentre essas avaliações temos a espirometria, que é um teste acurado e validado para avaliação da função pulmonar e diagnosticar os distúrbios ventilatórios. Para avaliação da força muscular respiratória, o teste padrão ouro é a manovacuometria, descrito desde 1999, com a primeira equação de $\mathrm{Neder}^{3}$ para o valor de predição da população brasileira, respalda até hoje, a avaliação respiratória na prática clínica. É um equipamento de fácil acesso, custobenefício, precisão em relação a determinação dos valores da pressão inspiratória (Pimáx) e expiratória máxima (Pemáx) $)^{4-6}$.

A Ventilação Voluntária Máxima (VVM) é um dos testes difundidos no cenário clínico, para avaliação da resistência da musculatura respiratória, mesmo sem ser validado para esta função. Nesta mensuração é avaliada a capacidade dos músculos respiratórios em mobilizar o maior volume de ar entre 10 a 15 segundos num esforço ventilatório voluntário máximo ${ }^{1,6}$.

A literatura ainda é controversa quanto a interpretação e aplicabilidade do uso da VVM na prática clínica. Um estudo realizado em pacientes com distrofia muscular miotônica, que analisaram a VVM como preditor para progressão da disfunção ventilatória. Sua hipótese foi de que a VVM é o primeiro parâmetro da função pulmonar que apresentou redução na população analisada. Eles confirmaram a teoria, contudo o teste de ventilação voluntária máxima ainda possui uma falta de especificidade quanto a sua real avaliação dos músculos respiratórios.

Além disso, durante os 12 segundos numa respiração sem carga pode não haver um estresse adequado dos músculos respiratórios, logo as informações são limitadas, e ainda não está elucidada a especificidade do teste. Assim, o objetivo desse estudo foi verificar a correlação a ventilação voluntária máxima com a força e resistência dos músculos respiratórios em jovens hígidos.

\section{Materiais e métodos}

Trata-se de um estudo observacional de corte transversal, que foi realizado na Clínica. Os voluntários foram recrutados no segundo semestre do ano de 2019, através do método Bola de $\mathrm{Neve}^{8}$. O estudo foi aprovado pelo comitê de ética de acordo com o seguinte CAAE 10849519.9.0000.5544. Os indivíduos que aceitaram participar da coleta, assinaram o termo de consentimento livre e esclarecido (TCLE), de acordo com a resolução 466/12. Esta metodologia foi guiada pelo STROBE? .

Foram incluídos indivíduos acima de 18 anos, ambos os sexos, hígidos, ativos e sedentários. Excluindo aqueles que apresentaram distúrbio ventilatório no exame espirométrico (restrição ou obstrução ventilatória), histórico de asma, tabagismo e falta de compreensão para realizar os testes. Os participantes foram instruídos a não ingerir álcool nas últimas 24 horas, não se exercitar no dia do exame e evitar roupas apertadas que potencialmente pudesse afetar sua dinâmica durante o teste².

Uma ficha de avaliação criada pela equipe pesquisadora foi realizada no primeiro momento, para coleta dos dados sociodemográficos, praticante de atividade física e o histórico clínico respiratório, e os resultados dos testes respiratórios.

\section{Avaliação da força dos músculos respiratórios}

A força muscular respiratória foi avaliada através do manovacuômetro sport analógico da marca

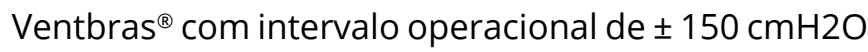
que é para obtenção da pressão inspiratória máxima (Pimáx) e pressão expiratória máxima (Pemáx). O bocal de tubo de borracha com diâmetro interno de 32 $\mathrm{mm}$ foi acoplado em uma peça de plástico que tem um diâmetro de aproximadamente dois $\mathrm{mm}$ de vazamento, que permitiu uma pequena quantidade de ar escapar para evitar qualquer ação dos músculos faciais. Os participantes foram orientados a manter-se em uma posição sentada, para realizar os movimentos respiratórios no equipamento, com as vias nasais ocluídas por um clip e os lábios selados em torno do bocal para que não ocorresse nenhum escape de ar. O equipamento foi calibrado e testado para garantir a confiabilidade dos dados coletados ${ }^{3}$. 
Os valores da Pimáx foram obtidos através de uma inspiração a partir do volume residual, repetindo pelo menos três vezes com um intervalo de um minuto entre as repetições. A mesma maneira aconteceu para a obtenção da Pemáx que ocorreu por uma expiração a partir da capacidade pulmonar total. $\mathrm{Na}$ manobra da Pimáx, o participante manteve o bocal na cavidade oral durante a inspiração e na Pemáx ao longo da expiração. $O$ valor adotado foi aquele mais alto em três repetições para cada manobra, sem ter a excedência de $10 \%$ do segundo valor mais alto, caso ocorresse era realizado uma quarta manobra. A manovacuometria foi realizada por um único avaliador, com as instruções verbais padronizadas, sempre no período da tarde e no mesmo local ${ }^{3}$.

\section{Avaliação da função pulmonar e ventilação vo- luntária máxima}

Para avaliação da função pulmonar e VVM foi adotado o protocolo da American Thoracic Society (ATS) e utilizado o Espirômetro FlowMax Pro $\AA^{2}$. O participante seguiu as orientações em manter-se na posição sentada, com a via nasal ocluída e os lábios selados em torno do bocal para que evitasse qualquer escape de ar durante o teste. Durante a avaliação, o participante recebeu a instrução de realizar uma inspiração máxima, seguida de uma expiração máxima por pelo menos seis segundos. Para realizar a VVM o indivíduo foi instruído a realizar a manobra de inspiração e expiração rápida e forçada durante 12 segundos. A medida foi repetida duas vezes após um período de recuperação de pelo menos cinco minutos, foi considerado à medida que obteve melhor desempenho ${ }^{1,2}$. O participante era informado com relação aos efeitos adversos (tontura, tosse e desmaio) que a técnica VVM poderia trazer e caso a pessoa sentisse qualquer um desses sintomas, o teste era interrompido.

\section{Avaliação da resistência dos músculos inspiratórios}

Para avaliação da resistência dos músculos respiratórios foi utilizado o teste de carga constante adaptado. O teste original é proposto com carga de $70 \%$ da Pimáx, porém foi realizado inicialmente um teste piloto, em que foi verificado que alguns voluntários permaneceram menos um minuto, desta forma usamos o teste com carga de $60 \%$ testado anteriormente por Ozalp e colaboradores ${ }^{10}$.
Foi utilizado o resistor de carga linear o equipamento da POWERBreath ${ }^{\circledR}$ Classic. Inicialmente o participante realizou aquecimento dos músculos inspiratórios com 30 repetições numa intensidade de 30\% da Pimáx, seguido de um repouso de dois minutos. Após esta fase, foi iniciado o teste com $60 \%$ da Pimáx, o voluntário foi incentivado a permanecer respirando sob esta carga o maior tempo possível. O teste foi interrompido quando o voluntário não conseguiu mais vencer a resistência imposta pelo dispositivo ${ }^{9}$. A variável de desfecho do teste foi o tempo de tolerância do voluntário respirando sob esta carga, denominado de tempo limite (Tlim). O ritmo respiratório foi controlado pelo pesquisador através de comando verbal, incentivando que voluntário realize uma inspiração a cada cinco segundos ${ }^{10}$.

\section{Análise estatística}

Para a análise dos dados foi utilizado o software Statistical Package for Social Sciences (SPSS) versão 14.0 para plataforma Windows. Para verificar a normalidade das variáveis numéricas realizou-se a estatística descritiva. As variáveis de distribuição simétrica (idade, massa, altura, IMC, número semanal da prática de atividade física, Pimáx, Pemáx, CVF, VEF1, PFE e VVM) foram apresentadas em média e desvio padrão e as assimétricas (tempo limite) em mediana e intervalo interquartil. Foi realizado teste t de Student para amostras independentes e Mann-Whitney para a variável tempo limite. Aplicamos a coeficiente de correlação de Pearson para a associação das variáveis Pimáx, Pemáx e VVM.

\section{Resultados}

A amostra foi composta por 27 participantes, com maior frequência do sexo masculino $59,3 \%$ e de ativos 55,6\%. A média de idade foi de $23,4 \pm 5,9$ anos e 0 IMC $23,6 \pm 3,0 \mathrm{~kg} / \mathrm{m}^{2}$. As características da população estão representadas na Tabela 1. 
Tabela 1. Dados sociodemográficos e antropométricos de 27 participantes hígidos. Salvador, Bahia, 2019.

\begin{tabular}{|c|c|c|}
\hline Variáveis & $\mathbf{n}$ & $\%$ \\
\hline \multicolumn{3}{|l|}{ Sexo } \\
\hline Masculino & 16 & 59,3 \\
\hline Feminino & 11 & 40,7 \\
\hline \multicolumn{3}{|l|}{ Atividade Física } \\
\hline Ativos & 15 & 55,6 \\
\hline \multirow[t]{2}{*}{ Sedentários } & 12 & 44,4 \\
\hline & média & DP \\
\hline Idade (anos) & 23,4 & $\pm 5,9$ \\
\hline Massa (kg) & 68,3 & $\pm 12,2$ \\
\hline Altura $(\mathrm{cm})$ & 169,6 & $\pm 7,4$ \\
\hline IMC $\left(\mathrm{kg} / \mathrm{m}^{2}\right)$ & 23,6 & $\pm 3,0$ \\
\hline
\end{tabular}

Todas as variáveis apresentadas, o sexo masculino apresentou valores médios mais elevados quando comparado com o feminino. Exceto, na relação VEF1/CVF, \% VEF1 e \% CVF em que as mulheres apresentaram as respectivas médias $87,0 \pm 5,7,97,8 \pm 5,9,97,8 \pm 5,9$ maior que a dos homens, com p não significante, Tabela 2.

Tabela 2. Dados espirometricos e da manovacuometria entre os grupos masculino e feminino em participantes hígidos. Salvador, Bahia, 2019

\begin{tabular}{|c|c|c|c|c|}
\hline Variáveis & & Média & DP & p \\
\hline \multirow[t]{2}{*}{ CVF } & Masculino & 4,5 & $\pm 0,5$ & $<0,001$ \\
\hline & Feminino & 3,5 & $\pm 0,3$ & \\
\hline \multirow[t]{2}{*}{$\%$ CVF } & Masculino & 96,6 & $\pm 8,1$ & 0,69 \\
\hline & Feminino & 97,8 & $\pm 5,9$ & \\
\hline \multirow[t]{2}{*}{$\mathrm{VEF}_{1}$} & Masculino & 3,9 & $\pm 0,4$ & $<0,001$ \\
\hline & Feminino & 3,0 & $\pm 0,3$ & \\
\hline \multirow[t]{2}{*}{$\%$ da VEF 1} & Masculino & 96,6 & $\pm 8,1$ & 0,69 \\
\hline & Feminino & 97,8 & $\pm 5,9$ & \\
\hline \multirow[t]{2}{*}{$\mathrm{VEF}_{1} / \mathrm{CVF}$} & Masculino & 86,4 & $\pm 4,5$ & 0,77 \\
\hline & Feminino & 87,0 & $\pm 5,7$ & \\
\hline \multirow[t]{2}{*}{ PEF } & Masculino & 8,5 & $\pm 1,5$ & $<0,001$ \\
\hline & Feminino & 6,0 & $\pm 1,4$ & \\
\hline \multirow{2}{*}{ VVM (L) } & Masculino & 132,9 & $\pm 28,4$ & 0,03 \\
\hline & Feminino & 100,5 & $\pm 18,8$ & \\
\hline \multirow[t]{2}{*}{ Predito VVM (L) } & Masculino & 164,7 & $\pm 18,7$ & $<0,001$ \\
\hline & Feminino & 131,8 & $\pm 13,5$ & \\
\hline \multirow[t]{2}{*}{ Pimáx $\left(\mathrm{cmH}_{2} \mathrm{O}\right)$} & Masculino & 112,8 & $\pm 24,9$ & 0,20 \\
\hline & Feminino & 99,5 & $\pm 27,8$ & \\
\hline \multirow[t]{2}{*}{ Pimáx $\left(\mathrm{cmH}_{2} \mathrm{O}\right)$ predita } & Masculino & 136,5 & $\pm 4,7$ & $<0,001$ \\
\hline & Feminino & 99,7 & $\pm 0,8$ & \\
\hline \multirow[t]{2}{*}{ Pemáx $\left(\mathrm{cmH}_{2} \mathrm{O}\right)$} & Masculino & 97,0 & $\pm 27,8$ & 0,02 \\
\hline & Feminino & 73,6 & $\pm 17,0$ & \\
\hline \multirow{3}{*}{ Pemáx $\left(\mathrm{cmH}_{2} \mathrm{O}\right)$ predita } & Masculino & 146,3 & $\pm 4,7$ & $<0,001$ \\
\hline & Feminino & 104,5 & $\pm 1,2$ & \\
\hline & & Mediana & I.Q & \\
\hline \multirow{2}{*}{ Tlim (segundos) } & Masculino & 170,0 & $(434,5-74,5)$ & 0,40 \\
\hline & Feminino & 213,0 & $(276-74,5)$ & \\
\hline
\end{tabular}

$\mathrm{DP}^{*}=$ desvio padrão. I.Q: Intervalo interquartil. Pimáx: pressão inspiratória máxima. Pemáx: pressão expiratória máxima. CVF: capacidade vital forçada. VVM: ventilação voluntária máxima. VEF1: volume expiratório forçado no primeiro segundo. M: Masculino. F: Feminino. Foi utilizado o teste $t$ student para amostras independentes e para variável Tlim foi utilizado teste Mann Whitney. 
A despeito dos praticantes de exercício físico, a CVF, VVM, Pimáx e Pemáx obtiveram respectivamente uma média de $4,2 \pm 0,7,130,3 \pm 28,3,121,2 \pm 20,7$ e $98,3 \pm 23,5$. Os valores foram maiores quando comparado com os sedentários, apresentando significância estatística. Por conseguinte, quando analisado o percentual do VEF1 e os preditos da VVM, os sedentários expressaram de modo respectivo as médias 97,9 $\pm 7,2$ e 151,6 623,0 . Assim como, na relação VEF1/CVF, os sedentários trouxeram uma média 89,0 $\pm 5,1$ maior que a dos ativos. Em referência ao tempo limite, os ativos tiveram uma média superior $244,4 \pm 300,3$, porém não houve significância, Tabela 3.

Tabela 3. Dados espirometricos e manovacuometria em indivíduos ativos e sedentários. Salvador, Bahia, 2019

\begin{tabular}{|c|c|c|c|c|}
\hline Variáveis & & Média & DP & p \\
\hline \multirow[t]{2}{*}{ CVF } & Ativo & 4,2 & $\pm 0,7$ & 0,05 \\
\hline & Sedentário & 4,0 & $\pm 0,7$ & \\
\hline \multirow[t]{2}{*}{$\%$ CVF } & Ativo & 96,5 & $\pm 7,3$ & 0,06 \\
\hline & Sedentário & 97,9 & $\pm 7,2$ & \\
\hline \multirow[t]{2}{*}{$\mathrm{VEF}_{1}$} & Ativo & 3,6 & $\pm 0,6$ & 0,09 \\
\hline & Sedentário & 3,6 & $\pm 0,6$ & \\
\hline \multirow[t]{2}{*}{$\%$ VEF $_{1}$} & Ativo & 96,5 & $\pm 7,3$ & 0,06 \\
\hline & Sedentário & 97,9 & $\pm 7,2$ & \\
\hline \multirow[t]{2}{*}{$\mathrm{VEF}_{1} / \mathrm{CVF}$} & Ativo & 84,8 & $\pm 4,1$ & 0,02 \\
\hline & Sedentário & 89,0 & $\pm 5,1$ & \\
\hline \multirow[t]{2}{*}{ PEF } & Ativo & 8,3 & $\pm 1,4$ & 0,02 \\
\hline & Sedentário & 6,5 & $\pm 2,0$ & \\
\hline \multirow[t]{2}{*}{ VVM (L) } & Ativo & 130,3 & $\pm 28,3$ & 0,03 \\
\hline & Sedentário & 106,5 & $\pm 26,0$ & \\
\hline \multirow{2}{*}{ Predito VVM (L) } & Ativo & 151,2 & $\pm 24,4$ & 0,09 \\
\hline & Sedentário & 151,6 & $\pm 23,0$ & \\
\hline \multirow[t]{2}{*}{ Pimáx $\left(\mathrm{cmH}_{2} \mathrm{O}\right)$} & Ativo & 121,2 & $\pm 20,7$ & 0,01 \\
\hline & Sedentário & 90,2 & $\pm 23,0$ & \\
\hline \multirow[t]{2}{*}{ Pimáx $\left(\mathrm{cmH}_{2} \mathrm{O}\right)$ predita } & Ativo & 123,5 & $\pm 18,4$ & 0,05 \\
\hline & Sedentário & 119,0 & $\pm 19,7$ & \\
\hline \multirow[t]{2}{*}{ Pemáx $\left(\mathrm{cmH}_{2} \mathrm{O}\right)$} & Ativo & 98,3 & $\pm 23,5$ & 0,01 \\
\hline & Sedentário & 74,0 & $\pm 24,0$ & \\
\hline \multirow[t]{3}{*}{ Pemáx $\left(\mathrm{cmH}_{2} \mathrm{O}\right)$ predita } & Ativo & 131,5 & $\pm 20,9$ & 0,05 \\
\hline & Sedentário & 126,4 & $\pm 22,2$ & \\
\hline & & Mediana & $\mathrm{I} . \mathrm{Q}$ & \\
\hline \multirow{2}{*}{ Tlim (segundos) } & Ativo & 136,5 & $(378,0-26,5)$ & 0,09 \\
\hline & Sedentário & 232,0 & $(271,0-135,0)$ & \\
\hline
\end{tabular}

Ao se verificar a correlação entre a VVM com a Pimáx e Pemáx, foi observada uma correlação moderada, e com significância estatística apresentando respectivamente $p=0,04$ e 0,02 e $r=0,53$ e 0,57, Figura 1 . 
Figura 1. Correlação entre as variáveis Pimáx, Pemáx e VVM em participantes hígidos. Correlação de Pearson. Salvador, Bahia, 2019.

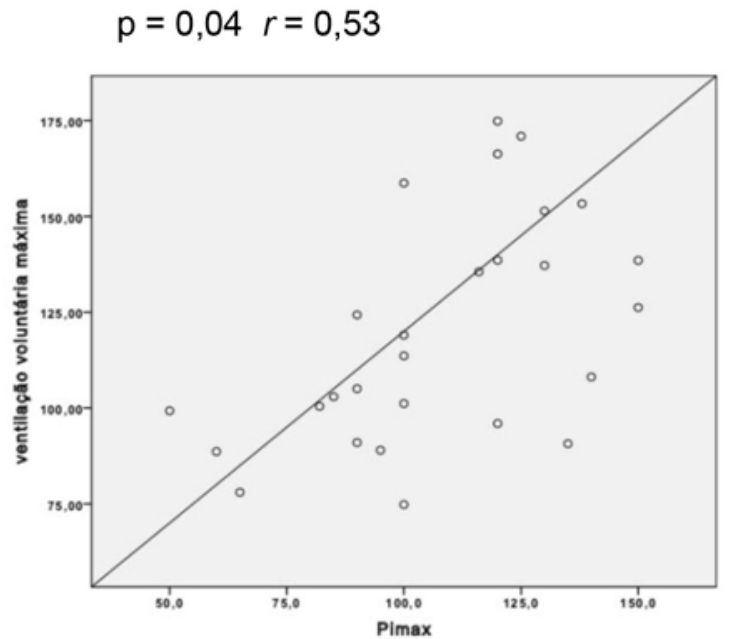

Discussão

Esta pesquisa é pioneira com a proposta de testar a correlação entre o teste de carga constante, a força dos músculos respiratórios com a ventilação voluntária máxima em voluntários hígidos. A partir da análise dos dados, identificamos que, a ventilação voluntária máxima apresenta melhor correlação com a força dos músculos respiratórios do que a resistência sob uma carga constante.

O método considerado padrão ouro para avaliar resistência dos músculos respiratórios é a hiperpneia voluntária máxima isocapnica. Neste método, o voluntário é estimulado a manter taxas de volume minuto num sistema fechado que permite manter valores equilibrados da pressão de dióxido de carbono $\left(\mathrm{PCO}_{2}\right)^{1,6}$. Entretanto, este teste necessita de um dispositivo de alto custo, consequentemente é pouco acessível na prática clínica brasileira. Diante disso, a VVM é utilizada para inferir a resistência e capacidade da musculatura ventilatória.

Todavia, a partir da análise dos resultados, sugere-se que a VVM é influenciada mais pela força dos músculos respiratórios do que pela resistência, haja vista que uma medida de curta duração e sem imposição de carga externa não configura uma medida de resistência dos músculos respiratórios. Entretanto, não é possível afirmar essa teoria nesse estudo. Já que não foi realizado o teste padrão ouro para avaliar a resistência.

É importante destacar que na amostra estudada, em concordância com a literatura ${ }^{13}$, se observa uma alta

$$
p=0,02 r=0,57
$$

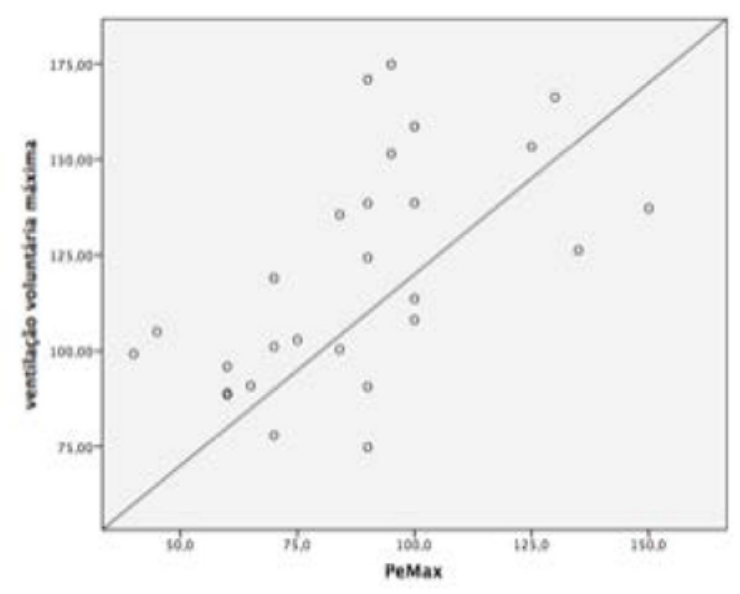

variação do tempo limite tolerado pelos voluntários respirando sob uma carga de $60 \%$ da Pimáx. Desta forma, destacamos a hipótese de que este teste não seja o mais sensível para identificar a baixa resistência dos músculos respiratórios. Neste estudo observamos que dois voluntários interromperam o teste por dor na ATM e um por dificuldade de deglutir saliva. Estes achados também foram encontrados por um grupo de pesquisadores do Brasil, que estudaram pacientes com DPOC ${ }^{13}$. Entretanto, se faz necessário, investigar esses sintomas, nas pesquisas que aplicam esse método de avaliação respiratória.

É essencial revelar que houve uma correlação moderada da força dos músculos inspiratórios e expiratórios com a VVM em jovens hígidos. Este achado pode ser explicado pelo fato de que na realização do teste o indivíduo é orientado a mobilizar uma grande quantidade de ar, num curto espaço de tempo ${ }^{1,6}$. Desta forma, recruta-se não só fibras de resistência, mas principalmente fibras do tipo $\mathrm{II}^{14}$, tornando a VVM um teste mais associado a potência muscular do que endurance.

Em relação ao sexo e as variáveis da função pulmonar e da força muscular respiratória, o sexo masculino apresentou médias mais altas, quando comparado com as mulheres. Estes achados são atribuídos a maior massa muscular, maior número de alvéolos e menor resistência das vias aéreas do sexo masculino $^{15}$. O aumento da carga resistiva nas mulheres é explicado pela diferença geométrica das vias aéreas entre os gêneros, resultando maior consumo de oxigênio dos músculos respiratórios nas mulheres ${ }^{14,15}$. 
Quando comparado os indivíduos ativos com os sedentários, foi comprovado que os ativos apresentaram maior VVM e força muscular. Em outro estudo, Oliveira e colaboradores ${ }^{16}$, não corroboraram com os resultados, em relação a força muscular respiratória. Essa discrepância pode ser explicada pelo tamanho amostral aplicada no estudo atual, além da predominância do sexo masculino, com médias mais altas quando associados com as mulheres.

As limitações advêm do método de avaliação da resistência, pois não foi utilizado o teste padrão ouro. Assim, o teste de carga constante deve ser interpretado com cautela.

\section{Conclusão}

O teste de ventilação voluntária máxima possui uma correlação moderada com a força muscular respiratória, e não obtém correlação com o teste de carga constante. Ademais, faz-se necessário outros estudos de se proponha a analisar outros testes de resistência da musculatura respiratória que seja de fácil acesso e útil na prática clínica.

\section{Agradecimentos}

Esse projeto foi financiado pela Fundação de Amparo a Pesquisa da Bahia (FAPESB), vinculado ao Grupo de Pesquisa de Fisioterapia Cardiovascular e Respiratória da Bahiana.

\section{Contribuições dos autores}

Oliveira FTO participou da orientação da pesquisa, concepção, estruturou o método do trabalho, delineamento, busca e análise estatística dos dados da pesquisa, coleta de dados, interpretação dos resultados, escrita do manuscrito, e revisão do artigo científico. Santos JG participou da concepção, delineamento, busca e análise estatística dos dados da pesquisa, coleta de dados, interpretação dos resultados, escrita do manuscrito e revisão do artigo científico. Dias CMCC participou da orientação da pesquisa, coordenação do grupo, concepção, delineamento, busca e análise estatística dos dados da pesquisa, coleta de dados, interpretação dos resultados, escrita do manuscrito e revisão do artigo científico. Ribeiro JR participou da coleta de dados, interpretação dos resultados, apresentou sugestões importantes, e revisão do artigo científico. Costa FAS participou da coleta de dados e apresentou sugestões para a pesquisa. Oliveira IAA participou da coleta de dados, interpretação dos resultados, apresentou sugestões importantes e redação do artigo científico. Almeida CN participou da coleta de dados e apresentou sugestões importantes.

\section{Conflitos de interesses}

Nenhum conflito financeiro, legal ou político envolvendo terceiros (governo, empresas e fundações privadas, etc.) foi declarado para nenhum aspecto do trabalho submetido (incluindo, mas não se limitando a subvenções e financiamentos, participação em conselho consultivo, desenho de estudo, preparação de manuscrito, análise estatística, etc.).

\section{Referências}

1. Laveneziana $P$, Albuquerque $A$, Aliverti $A, B a b b T$, Barreiro $\mathrm{E}$, Dres $\mathrm{M}$ et al.ERS statement on respiratory muscle testing at rest and during exercise. Eur Respir J. 2019;53:1801214. doi: 10.1183/13993003.01214-2018

2. Miller MR, Hankinson J, Brusasco V, Burgos F, Casaburi R, Coates A et al. Standardisation of spirometry. Eur Respir J. 2005;26(2):319-38. doi: 10.1183/09031936.05.00034805

3. Neder JA, Andreoni S, Lerario MC, Nery LE. Reference values for lung function tests. II. Maximal respiratory pressures and voluntary ventilation. Brazilian J Med Biol Res. 1999;32(6):719-27. doi: 10.1590/S0100-879X1999000600007

4. Costa D, Gonçalves HA, Lima LP, Ike D, Cancelliero KM, Montebelo MIL. Novos valores de referência para pressões respiratórias máximas na população brasileira.J Bras Pneumol. 2010;36(3):306-12. doi: 10.1590/\$1806-37132010000300007

5. Simões RP, Deus APL, Auad MA, Dionísio J, Mazzonetto M, Borghi-Silva A. Maximal respiratory pressure in healthy 20 to 89 year-old sedentary individuals of central São Paulo State. Rev Bras Fisioter. 2010;14(1):60-7. doi: 10.1590/S1413-35552010000100010

6. Silva PE, Chiappa GR, Vieira PJC, Roncada C. Avaliação da função muscular ventilarória. PROFISIO - Fisioter Cardiovasc e Respir. 2016;3(April):9-46.

7. Suh MR, Kim DH, Jung J, Kim B, Lee JW, Choi WA et al. Clinical implication of maximal voluntary ventilation in myotonic muscular dystrophy. Medicine (Baltimore). 2019;98(18):e15321. doi: 10.1097/MD.0000000000015321

8. Vinuto J. A amostragem em bola de neve na pesquisa qualitativa: um debate em aberto. Temáticas. 2016;22(44):203-20.

9. Malta M, Cardoso LO, Bastos Fl, Magnanini MMF, Silva CMFP. Iniciativa STROBE: subsídios para a comunicação de estudos observacionais. Rev Saude Publica. 2010;44(3):559-65. doi: 10.1590/S0034-89102010000300021

10. Ozalp O, Inal-Ince D, Cakmak A, Calik-Kutukcu E, Saglam $M$, Savci $S$ et al. High-intensity inspiratory muscle training in bronchiectasis: A randomized controlled trial. Respirology. 2019;24(3):246-53. doi: 10.1111/resp.13397 
11. Hulley SB, Cummings SR, Browner WS, Grady DG, Thomas B, Newman TB. Delineando a pequisa clínica. 4.ed. Artmed; 2015.

12. Jurić I, Labor S, Plavec D, Labor M. Inspiratory muscle strength affects anaerobic endurance in professional athletes. Arh Hig Rada Toksikol. 2019;70(1):42-8. doi: 10.2478/aiht-2019-70-3182

13. Basso-Vanelli RP, Di Lorenzo VAP, Ramalho M, Labadessa IG, Regueiro EMG, Jamami M et al. Reproducibility of inspiratory muscle endurance testing using PowerBreathe for COPD patients. Physiother Res Int. 2018;23(1):1-6. doi: 10.1002/pri.1687

14. Aliverti A. The respiratory muscles during exercise. Breathe. 2016;12(2):165-8. doi: 10.1183/20734735.008116

15. Sheel AW, Dominelli PB, Molgat-Seon Y. Revisiting dysanapsis: Sex-based differences in airways and the mechanics of breathing during exercise. Exp Physiol. 2016;101(2):213-8. doi: 10.1113/ EP085366

16. Oliveira FTO, Petto J, Esquivel MS, Dias CMCC, Oliveira ACS, Aras R. Comparação da força e resistência dos músculos inspiratórios entre ativos e sedentários. Rev Pesq Fisio. 2018;8(2):223-9. doi: 10.17267/2238-2704rpf.v8i2.1926 